\title{
AHP-Based Classifier Combination
}

\author{
László Felföldi \\ Department of Informatics, University of Szeged \\ H-6720 Szeged, Árpád tér 2., Hungary \\ lfelfold@inf.u-szeged.hu \\ András Kocsor \\ Research Group on Artificial Intelligence of the Hungarian Academy of Sciences and University of Szeged \\ H-6720 Szeged, Aradi vértanúk tere 1., Hungary \\ kocsor@inf.u-szeged.hu
}

Key words: Classifier combination, Analytic hiearchy process, supervised learning

\begin{abstract}
Classifier combinations are effective techniques for difficult pattern recognition problems such as speech recognition where the combination of differently trained classifiers can produce a more robust phoneme classification on noisy datasets. In this paper we investigate traditional linear combination schemes (e.g. arithmetic mean and least squares methods), and propose a new combiner based on the Analytic Hierarchy Process (AHP), a method frequently applied in mathematical psychology and multi-criteria decision making. In addition, we experimentally compare the applicability of these linear combination schemes using neural network classifiers on a speech recognition framework and two test sets from the UCI repository.
\end{abstract}

\section{Introduction}

In pattern recognition problems (Jain et al., 2000)(Vapnik, 1998)(Duda et al., 2001) the main aim is to construct a classifier (or inducer) in order to model the behavior of a system. For each pattern of a pattern space the classifier has to select a class label from the set of available labels. The construction of a classifier (i.e. the learning process) is based on a set of labelled examples, and strongly depends on prior knowledge about the pattern space and the characteristics of the given examples. Given infinite training data, consistent classifiers approximate the Bayesian decision boundaries to arbitrary precision, therefore providing a similar generalization. However, often only a limited portion of the pattern space is available or observable. Given a finite and noisy data set, different classifiers typically provide different generalizations. It is thus necessary to train more networks when dealing with classification problems to ensure that a good model or parameter set is found. However, selecting such a classifier is not necessarily the ideal choice since potentially valuable information may be wasted by discarding the results of the other classifiers. In order to avoid this kind of loss of information, the output of all available classifiers should be examined for the final decision. This approach is particularly useful for difficult problems such as those that involve a large amount of noise.
A fair number of combination methods have been proposed in the literature (Xu et al., 1992) which have proved effective in improving classifier performance. In this paper we focus on linear combination schemes, especially averaging techniques. Experimental studies have shown that linear classifier combinations can improve the recognition accuracy. Tumer and Ghosh showed that combining networks using single averaging reduces the variance of the actual decision boundaries around the optimum boundary (Tumer and Ghosh, 1996). Later Fumera and Roli extended the theoretical framework for the weighted averaging rule (Roli and Fumera, 2002). However, these theoretical explanations apply very restrictive assumptions, hence they cannot show the real performance of the combiner. To improve the accuracy of the averaging techniques, we propose a new combination method based on the Analytical Hierarchy Process, and demontrate that it may produce a more robust classification performance in tests

The paper is organized as follows. In the next section we give a brief overview of linear combination schemes, including averaging techniques, and examine the theoretical background of these systems. The third section introduces the Analytic Hierarchy Process and shows utilization facilities provided in combination frameworks. The experimental section then compares the performance of various linear schemes on different database and classifier sets. Finally, we 
give some brief conclusions and ideas for future research.

\section{Linear Classifier Combinations}

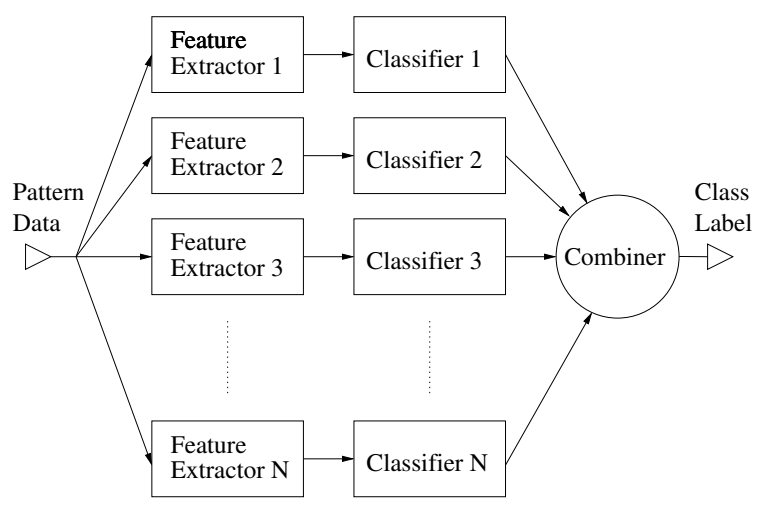

Figure 1: General parallel combination scheme

Combiners aggregate the outputs of different classifiers to make a final decision. This aggregation depends on the kind of information that the individual classifiers can supply. From this viewpoint the classification methods can be grouped into three main categories:

1. Measurement or Confidence. The classifier can model probability values for each class label. Let $f_{i}^{j}(x)$ denote the output of classifier $\mathcal{C}_{j}$ for class $i$ and pattern $x$. The linear combination method can be described by the formula

$$
\hat{f}_{i}(x)=\sum_{j=1}^{N} w_{j} f_{i}^{j}(x),
$$

where $\hat{f}_{i}(x)$ is the combined class conditional probability, and $w_{j}$ is the weighting factor of classifier $\mathcal{C}_{j}$. The final decision can be obtained by selecting the class with the greatest probability, in accordance with the Bayesian decision principle.

2. Ranking. The classifier can produce a list of class labels in the order of their probabilities. The combined position $\hat{g}_{i}$ is then computed via the formula

$$
\hat{g}_{i}(x)=\sum_{j=1}^{N} w_{j} g_{i}^{j}(x),
$$

where $g_{i}^{j}(x)$ is the position of the label $i$ in the classification result of pattern $x$ obtained by the classifier $\mathcal{C}_{j}$. With the selection of a proper monotonic decreasing function $u$, and

$$
f_{i}^{j}(x)=u\left(g_{i}^{j}(x)\right),
$$

the ranking-type combination can be reduced to the confidence-type scheme.

3. Abstract. The classifier supplies only the most probable class label. In this case the combination relies on the majority voting formula

$$
\hat{\mathcal{L}}(x)=\arg \max _{i} \sum_{\mathcal{L}_{j}(x)=i} w_{j},
$$

where $\mathcal{L}_{j}(x)$ is the index of the class label calculated for pattern $x$. Taking the selection of $f_{i}^{j}(x)$ as

$$
f_{i}^{j}(x)= \begin{cases}1 & \mathcal{L}_{j}(x)=i \\ 0 & \text { otherwise }\end{cases}
$$

leads to the reformulation of voting as a confidence-type combination much like that for the ranking type.

As we have shown, the output of classifiers belonging to the Ranking or Abstract class can be transformed to class conditional probability values. Therefore, in the following section we shall deal only with the confidence-type combination, and expect the combiners to supply the class conditional probabilities.

We commence our study of linear combinations with a theoretical investigation, and then show how to apply them in practice.

\subsection{Theoretical investigation}

As mentioned previously, the output of the classifiers are expected to approximate the corresponding a posteriori probabilities if they are reasonably well trained. Thus the decision boundaries obtained using this kind of classifier are close to Bayesian decision boundaries. Tumer and Ghosh developed a theoretical framework for analyzing the averaging rule of linear combinations (Tumer and Ghosh, 1996). Later Roli and Fumera extended this concept by examining the weighted averaging rule (Roli and Fumera, 2002).

We shall focus on the classification performance near these decision boundaries. Consider the boundary between class $i_{1}$ and $i_{2}$. The output of the classifier is

$$
f_{i}(x)=p_{i}(x)+\epsilon_{i}(x)
$$

where $p_{i}(x)$ is the real a posteriori probability of class $i$, and $\epsilon_{i}(x)$ is the estimation error. Let us assume that the class boundary $x_{b}$ obtained from the approximated a posteriori probabilities

$$
f_{i_{1}}\left(x_{b}\right)=f_{i_{2}}\left(x_{b}\right)
$$

are close to the Bayesian boundaries $x^{*}$, i.e.

$$
p_{i_{1}}\left(x^{*}\right)=p_{i_{2}}\left(x^{*}\right),
$$


for boundary between class $i_{1}$ and $i_{2}$. Additionally assuming that the estimated errors $\epsilon_{i}(x)$ on different classes are independently and identically distributed (i.i.d.) variables with zero mean, Tumer and Ghosh showed that the expected error of classification can be expressed as:

$$
E=E_{\text {Bayes }}+E_{a d d},
$$

where $E_{\text {Bayes }}$ is the error of a classifier with the correct Bayesian boundary. The added error $E_{a d d}$ can be expressed as:

$$
E_{a d d}=\frac{s \sigma_{b}^{2}}{2},
$$

where $\sigma_{b}^{2}$ is the variance of $b$,

$$
b=\frac{\epsilon_{i_{1}}\left(x_{b}\right)-\epsilon_{i_{2}}\left(x_{b}\right)}{s},
$$

and $s$ is a constant term depending on the derivatives of the probability density functions at the optimal decision boundary.

Let us consider the effect of combining multiple classifiers. We shall deal only with linear combinations, so we have the following combined probabilities:

$$
\hat{f}_{i}(x)=\sum_{j=1}^{N} w_{j} f_{i}^{j}(x),
$$

where $f_{i}^{j}$ denotes the output of the classifier $\mathcal{C}_{j}$ for the class $i$. Assuming normalized weights, i.e.

$$
\sum_{j=1}^{N} w_{j}=1, w_{j} \geq 0
$$

we have that

$$
\hat{f}_{i}(x)=p_{i}(x)+\hat{\epsilon}_{i}(x)
$$

where

$$
\hat{\epsilon}_{i}(x)=\sum_{j=1}^{N} w_{j} \epsilon_{i}^{j}(x)
$$

Let us compute the variance of $\hat{b}$, where

$$
\hat{b}=\frac{\hat{\epsilon}_{i_{1}}-\hat{\epsilon}_{i_{2}}}{s} .
$$

Assuming that $\epsilon_{i}^{j}(x)$ are i.i.d. variables with zero mean and variance $\sigma_{\epsilon^{j}}^{2}$, the errors of different classifiers on the same class are correlated, while on different classes they are uncorrelated.

$$
\operatorname{Cov}\left(\epsilon_{i_{1}}^{m}\left(x_{b}\right), \epsilon_{i_{2}}^{n}\left(x_{b}\right)\right)=\left\{\begin{array}{ll}
\rho_{m n} & \text { when } i_{1}=i_{2} \\
0 & \text { otherwise }
\end{array},\right.
$$

where $\rho_{m n}$ denotes the covariance between the errors of classifier $\mathcal{C}_{m}$ and $\mathcal{C}_{n}$ for each class. Expanding the $\operatorname{tag} \sigma_{\epsilon}^{2}$ in Eq. (10), we find that

$$
\begin{aligned}
\hat{E}_{a d d} & =\frac{1}{s} \sum_{j=1}^{N} w_{j}^{2} \sigma_{\epsilon^{j}}^{2} \\
& +\frac{1}{s} \sum_{m \neq n} w_{m} w_{n} \rho_{m n} .
\end{aligned}
$$

Expressed in terms of additional errors of the classifiers

$$
\begin{aligned}
\hat{E}_{a d d} & =\frac{1}{s} \sum_{j=1}^{N} w_{j}^{2} E_{a d d}^{j} \\
& +\frac{1}{s} \sum_{m \neq n} w_{m} w_{n} \rho_{m n}
\end{aligned}
$$

where the term $E_{a d d}^{j}$ denotes the added error of the classifier $\mathcal{C}_{j}$. In the case of uncorrelated estimation errors (i.e. when $\rho_{m n}=0$ ), this equation reduces to

$$
\hat{E}_{a d d}=\sum_{j=1}^{N} w_{j}^{2} E_{a d d}^{j}
$$

which leads to the following optimal values for $w$ :

$$
w_{j}=c \frac{1}{E_{a d d}^{j}},
$$

where $c$ is a normalization factor. With equally performing classifiers, that is when all the errors $E_{a d d}^{j}$ have the same value

$$
E_{a d d}^{j}=E_{a d d}, j=1, \ldots, N,
$$

we obtain the Simple Averaging rule:

$$
w_{j}=\frac{1}{N}
$$

which results in the following error value:

$$
\hat{E}_{a d d}=\frac{1}{N} E_{a d d} .
$$

This formula shows that, under the conditions mentioned, linear combinations reduce the error of the individual classifier. Considering correlated errors, however, does not lead to a simple general expression for optimal values of weights, and other methods are required to estimate the optimal parameters.

\subsection{Construction}

To achieve the best combination performance the parameters of the combiner can be trained on a selected training data set. The form of linear combinations we 
deal with is quite simple (see Figure 1), the trainable parameters being just the weights of classifiers. Thus the various linear combinations differ only in the values of these factors. In the following we give some examples of methods commonly employed, and in the next section we propose a new method for computing these parameters.

1. Simple Averaging. In this simplest case, the weights can be selected so they have the same value:

$$
w_{j}=\frac{1}{N} \text {. }
$$

As mentioned before, this selection is optimal when all the classifiers have a very similar performance, and all the assumptions mentioned in Section 2.1 hold.

2. Weighted Averaging. Experiments show (Roli and Fumera, 2002) that Simple Averaging can be outperformed when selecting weights to be inversely proportional to the error rate of the corresponding classifier:

$$
w_{j}=\frac{1}{E_{j}},
$$

where $E_{j}$ is the error rate of the classifier $\mathcal{C}_{j}$, i.e. the ratio of the number of correctly classified patterns and total number of patterns on a selected data set for training the combiner. This rule (a more general version of the Simple Averaging rule) is employed in order to handle the combination of unequally performing classifiers.

3. Least-squares methods. To calculate the weights $w_{j}$ one can take those values that minimize the distance between the computed and estimated class conditional probabilities:

$$
\min _{w} \sum_{x \in \mathcal{X}} \sum_{i=1}^{l}\left(\sum_{j=1}^{N} w_{j} f_{i}^{j}(x)-p_{i}(x)\right)^{2}
$$

where

$$
\mathcal{X}=\left\{x_{1}, \ldots, x_{m}\right\}
$$

is the training set of patterns for the combination, and $p_{i}(x)$ is the estimated class conditional probability function. In the case of supervised learning the class labels are available for all the training patterns, but there is no direct way of comparing this information with computed a posteriori probabilities. Assuming a Bayesian decision, the pattern $x$ has the label $\mathcal{L}(x)=i$ when $p_{i}(x) \geq p_{j}(x)$ for all $i \neq j$. Let us approximate $p_{i}(x)$ by

$$
p_{i}(x)=\left\{\begin{array}{ll}
1 & \text { if } \mathcal{L}(x)=i \\
0 & \text { otherwise }
\end{array},\right.
$$

or calculate it from the error correlation matrix of the $n$ combiners (Perrone and Cooper, 1993). Using matrix notations the problem described in Eq.
(24) can be expressed as:

$$
\min _{w}(A w-b)^{T}(A w-b)
$$

where

$$
\begin{gathered}
a_{j,(m i+k)}=f_{i}^{j}\left(x_{k}\right), \\
b_{m i+k}=p_{i}\left(x_{k}\right),
\end{gathered}
$$

This optimization problem leads to the following linear equation:

$$
A^{T} A w=A^{T} b,
$$

which provides a way of determining the weighting factors.

\section{The Analytic Hierarchy Process}

Analytic Hierarchy Process is an intuitive and efficient method for multi-criteria decision-making (MCDM) applications (Saaty, 1980). The structure of a typical decision problem (see Fig. 2) consists of alternatives and decision criteria. Each alternative can be evaluated in terms of the decision criteria and, in the case of multilevel criteria, the relative importance (or weight) of each criterion can also be estimated.

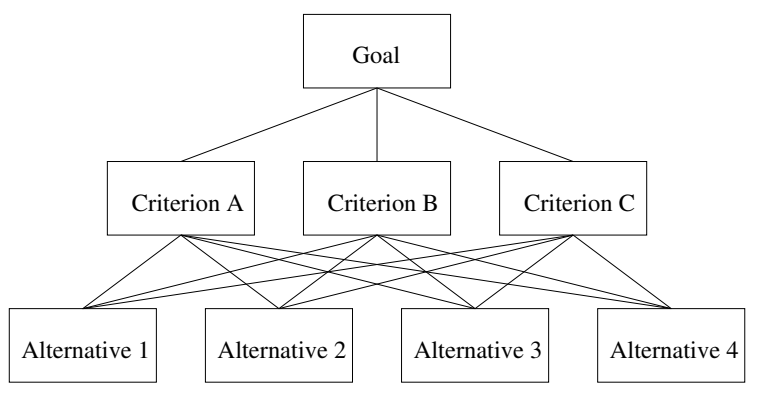

Figure 2: A simple application of AHP

In the following we give a brief summary of the fundamental properties of the AHP, and propose a combination method based on it.

\subsection{Mathematical model}

The first step of AHP is to divide the decision problem into sub-problems, which are structured into hierarchy levels. The number of levels depends on the complexity of the initial problem. The leaves contain the possible alternatives and the inner nodes represent the criteria. To compute the importance of possible choices, pairwise comparison matrices are utilized for each criterion. The element $a_{i j}$ of the comparison matrix $A$ represents the relative importance of choice $i$ 
against the choice $j$, implying that the element $a_{j i}$ is the reciprocal of $a_{i j}$. Let the importance value $v$ of choice $y$ be expressed as a linear combination of the importance values for each applied criterion:

$$
v(y)=\sum_{j=1}^{n} w_{j} v\left(y_{j}\right),
$$

where $w_{j}$ is the importance of choice $y$ with respect to the criterion $y_{j}$. Using comparison matrices AHP propagates the importance values of each node from the topmost criteria towards the alternatives, and selects the alternative with the greatest importance value as its final decision.

Let us now focus on the computation of the weights $w$ for a selected criterion. The elements of a given pairwise comparison matrix approximate the relative importance of the choices, thus

$$
a_{i j} \approx \frac{w_{i}}{w_{j}}
$$

where the elements of the unknown vector $w$ are the importance values. A matrix $M$ is called consistent if its components satisfy the following equalities:

$$
m_{i j}=\frac{1}{m_{j i}},
$$

and

$$
m_{i j}=m_{i k} m_{k j} \forall i, j, k .
$$

If $A$ is not consistent, it is not possible to find a vector $w$ that satisfies the equation

$$
a_{i j}=\frac{w_{i}}{w_{j}} .
$$

Now let us define the matrix of weight ratios by

$$
W=\left(\begin{array}{ccccc}
\frac{w_{1}}{w_{1}} & \frac{w_{1}}{w_{2}} & \frac{w_{1}}{w_{3}} & \ldots & \frac{w_{1}}{w_{n}} \\
\frac{w_{2}}{w_{1}} & \frac{w_{2}}{w_{2}} & \frac{w_{2}}{w_{3}} & \ldots & \frac{w_{2}}{w_{n}} \\
\frac{w_{3}}{w_{1}} & \frac{w_{3}}{w_{2}} & \frac{w_{3}}{w_{3}} & \ldots & \frac{w_{3}}{w_{n}} \\
\vdots & \vdots & \vdots & \ddots & \vdots \\
\frac{w_{n}}{w_{1}} & \frac{w_{n}}{w_{2}} & \frac{w_{n}}{w_{3}} & \cdots & \frac{w_{n}}{w_{n}}
\end{array}\right)
$$

or, in matrix notation,

$$
W=w w^{T} .
$$

Note that Eqs. (30) and (31) hold for the matrix $W$ :

$$
w_{i j}=\frac{w_{i}}{w_{j}}=\frac{w_{i}}{w_{k}} \frac{w_{k}}{w_{j}}=w_{i k} w_{k j},
$$

hence the matrix of weight ratios is consistent.

Because the rows of matrix $W$ are linearly dependent, the rank of the matrix is 1 , and there is only one nonzero eigenvalue. Knowing that the trace of a matrix is invariant under similarity transformations, the sum of diagonal elements is equal to the sum of eigenvalues, which implies that the nonzero eigenvalue $\lambda_{\max }$ equals the number of the rows:

$$
\lambda_{\max }=n \text {. }
$$

It is straightforward to check that the vector $w$ is an eigenvector of matrix $W$ corresponding to the maximum eigenvalue

$$
\begin{aligned}
(W w)_{i} & =\sum_{j=1}^{n} W_{i j} w_{j} \\
& =\sum_{i=1}^{n} \frac{w_{i}}{w_{j}} w_{j}=\sum_{j=1}^{n} w_{i} \\
& =n w_{i} .
\end{aligned}
$$

The aim of AHP is to resolve the weight vector $w$ from a pairwise comparison matrix $A$, where the elements of $A$ corresponds to the measured or estimated weight ratios. Following Saaty we shall assume that

$$
a_{i j}>0
$$

and

$$
a_{i j}=\frac{1}{a_{j i}} .
$$

From matrix theory it is known that a small perturbation of the coefficients implies a small perturbation of the eigenvalues. Hence we still expect to find an eigenvalue close to $n$, and select the elements of the corresponding eigenvector as weights. It can be proved that

$$
\lambda_{\max } \geq n,
$$

and the matrix $A$ is consistent if and only if $\lambda_{\max }=$ $n$. A way of measuring the consistency of the matrix $A$ is by defining the consistency index $(C I)$ as the negative average of the remaining eigenvalues:

$$
C I=\frac{\sum_{\lambda<\lambda_{\max }} \lambda}{n-1}=\frac{\lambda_{\max }-n}{n-1}
$$

\subsection{Combination using AHP}

As mentioned above, AHP provides the following solution for the problem of linear MCDM systems:

$$
v(y)=\sum_{i=1}^{n} w_{i} v\left(y_{i}\right)
$$

where the importance value of the choice is the linear combination of the importance values of the direct criteria. In linear classifier combinations the combined class conditional probabilities are computed as weighted sums of the probability values from each classifier, so

$$
f_{i}(x)=\sum_{j=1}^{N} w_{j} f_{i}^{j}(x) .
$$


Noting the similarities between these two methods, it is clear that, by applying pairwise comparisons on classifiers performance, AHP provides a way of computing the weights of inducers in classifier combinations. Let us calculate the element $a_{i j}$ of the comparison matrix as the quotient of classification performance on a selected test data set:

$$
a_{i j}=\frac{\frac{1}{E_{i}}}{\frac{1}{E_{j}}}=\frac{1}{a_{j i}},
$$

where $E_{i}$ is the classification error of classifier $\mathcal{C}_{i}$. If all the performance errors are measured on the same test data set, the comparison matrix $A$ is consistent, and the elements of the eigenvector whose corresponding eigenvalue is $N$, that is

$$
w_{i}=\frac{1}{E_{i}},
$$

are the same those as generated by weighted averaging. However, this method allows us to make pairwise comparisons of different inducers applied on different (e.g. randomly generated) test sets, taking advantage of the stabilizing effect of AHP. This leads to more a robust classification performance, especially in noisy environments, as shown in the experimental section.

\section{Experiments}

In this section we describe our experiments for comparing the performance of averaging combiners and our AHP-based combiner.

\subsection{Evaluation Domain}

In the experiments three data sets were employed: a data-set used in our speech recognition system, and two other datasets (letter and satimage) originating from the statlog/UCI repository. (http://www.liacc.up.pt/ML/statlog)

1. Speech data set (Kocsor and Tóth, 2003). The database is based on recorded samples taken from 160 children aged between 6 and 8 . The ratio of girls and boys was 50\% - 50\%.The speech signals were recorded and stored at a sampling rate of $22050 \mathrm{~Hz}$ in 16-bit quality. Each speaker uttered all the Hungarian vowels, one after the other, separated by a short pause. Since we decided not to discriminate their long and short versions, we only worked with 9 vowels altogether. The recordings were divided into a train and a test set in a ratio of $50 \%-50 \%$. There are numerous methods for obtaining representative feature vectors from speech data, but their common property is that they are all extracted from $20-30$ ms chunks or frames of the signal in 5-10 ms time steps. The simplest possible feature set consists of the so-called barkscaled filterbank log-energies (FBLE). This means that the signal is decomposed with a special filterbank and the energies in these filters are used to parameterize speech on a frame-by-frame basis. In our tests the filters were approximated via Fourier analysis with a triangular weighting. Altogether 24 filters were necessary to cover the frequency range from 0 to $11025 \mathrm{~Hz}$. Although the resulting log-energy values are usually sent through a cosine transformation to obtain the well-known melfrequency cepstral coefficients (MFCC), we abandoned it because, as we observed earlier, the learner we work with is not sensitive to feature correlations so a cosine transformation would bring no significant improvement.

2. Letter Data Set. The objective of the Data set is to identify each of a large number of black-and-white rectangular pixel displays as one of the 26 capital letters in the English alphabet. The character images were based on 20 different fonts and each letter within these 20 fonts was randomly distorted to produce a file of 20,000 unique stimuli. Each stimulus was converted into 16 primitive numerical attributes (statistical moments and edge counts) which were then scaled to fit into a range of integer values from 0 to 15 . We typically trained on the first 16000 items and then used the resulting model to predict the letter category for the remaining 4000 .

3. Satimage Data Set. One frame of Landsat MSS imagery consists of four digital images of the same scene in different spectral bands. Two of these are in the visible region (corresponding approximately to green and red regions of the visible spectrum) and two are in the (near) infra-red. Each pixel is a 8-bit binary word, with 0 corresponding to black and 255 to white. The spatial resolution of a pixel is about $80 \mathrm{~m} \times 80 \mathrm{~m}$. Each image contains $2340 \mathrm{x}$ 3380 such pixels. The database is a (tiny) sub-area of a scene, consisting of $82 \times 100$ pixels. Each line of data corresponds to a $3 \times 3$ square neighborhood of pixels completely contained within the $82 \times 100$ sub-area. Each line contains the pixel values in the four spectral bands (converted to ASCII) of each of the 9 pixels in the $3 \times 3$ neighborhood and a number indicating the classification label of the central pixel. The number of possible class labels for each pixel is 7 . We trained on the first 4435 patterns of the database and selected the remaining 2000 patterns for testing. 


\subsection{Evaluation Method}

During the experiments we compared the performance of 6 different combiners applied on each of the 3 databases. For each of the databases we trained 3layered neural networks with different structures, and selected 5 subsets of classifiers, denoted by Set 1 to Set5.

In the case of the speech and letter databases we trained networks setting the number of neurons in the hidden layer to $5,10,20,40,80,160$, and 320 . Table 1 shows the construction of classifier sets. The columns refer to the number of hidden neurons, and the rows show which networks belong to the selected classifier sets.

\begin{tabular}{c||c|c|c|c|c|c|c} 
& 5 & 10 & 20 & 40 & 80 & 160 & 320 \\
\hline \hline Set1 & $\mathrm{x}$ & $\mathrm{x}$ & $\mathrm{x}$ & $\mathrm{x}$ & $\mathrm{x}$ & $\mathrm{x}$ & $\mathrm{x}$ \\
\hline Set2 & & $\mathrm{x}$ & $\mathrm{x}$ & $\mathrm{x}$ & $\mathrm{x}$ & $\mathrm{x}$ & $\mathrm{x}$ \\
\hline Set3 & & & $\mathrm{x}$ & $\mathrm{x}$ & $\mathrm{x}$ & $\mathrm{x}$ & $\mathrm{x}$ \\
\hline Set4 & & & & $\mathrm{x}$ & $\mathrm{x}$ & $\mathrm{x}$ & $\mathrm{x}$ \\
\hline Set5 & & & & $\mathrm{x}$ & $\mathrm{x}$ & $\mathrm{x}$ &
\end{tabular}

Table 1: Classifier sets for the speech and letter databases.

The satimage database contains only 7 classes. In this case we trained networks with hidden layer size selected to $2,5,10,20,40,80$, and 160 . The corresponding classifier selection is displayed in Table 2.

\begin{tabular}{c||c|c|c|c|c|c|c} 
& 2 & 5 & 10 & 20 & 40 & 80 & 160 \\
\hline \hline Set1 & $\mathrm{x}$ & $\mathrm{x}$ & $\mathrm{x}$ & $\mathrm{x}$ & $\mathrm{x}$ & $\mathrm{x}$ & $\mathrm{x}$ \\
\hline Set2 & & $\mathrm{x}$ & $\mathrm{x}$ & $\mathrm{x}$ & $\mathrm{x}$ & $\mathrm{x}$ & $\mathrm{x}$ \\
\hline Set3 & & & $\mathrm{x}$ & $\mathrm{x}$ & $\mathrm{x}$ & $\mathrm{x}$ & $\mathrm{x}$ \\
\hline Set4 & & & & $\mathrm{x}$ & $\mathrm{x}$ & $\mathrm{x}$ & $\mathrm{x}$ \\
\hline Set5 & & & & $\mathrm{x}$ & $\mathrm{x}$ & $\mathrm{x}$ &
\end{tabular}

Table 2: Classifier sets for the satimage database.

The experiments compared the performance of linear combination schemes with different methods for acquiring the proper weights. We examined 2 schemes of Averaging : $S A$ and $W A$ (i.e. simple and weighted) averaging, and 4 schemes of $A H P$. To calculate the pairwise comparison matrices needed for the AHP method, we took the quotient of classification errors of the two competing networks on a random test set generated by bootstrapping (resampling the training data set with replacement) of the training set. In accordance with the size of the generated test set we had 4 AHP schemes, $A H P 1$ to $A H P 4$, setting the size of each to 50,100, 200 and 400, respectively. With the $W A$ combiner, the original training set was selected for the calculation of the weights.

\subsection{Results and Discussion}

\begin{tabular}{|l||c|c|c|r|r|}
\hline & Set1 & Set2 & Set3 & Set4 & Set5 \\
\hline \hline$S A$ & $\mathbf{8 . 5 2}$ & $\mathbf{9 . 2 6}$ & 9.95 & 9.77 & 8.80 \\
\hline$W A$ & 8.66 & 9.21 & 9.91 & 9.81 & 8.75 \\
\hline$A H P_{1}$ & 8.66 & 8.94 & $\mathbf{9 . 4 4}$ & 10.05 & 8.80 \\
\hline$A H P_{2}$ & 8.56 & 9.12 & 9.72 & 10.19 & 8.80 \\
\hline$A H P_{3}$ & 8.61 & 9.21 & 9.49 & 9.58 & $\mathbf{8 . 7 0}$ \\
\hline$A H P_{4}$ & 8.61 & 9.31 & 9.55 & $\mathbf{9 . 0 7}$ & $\mathbf{8 . 7 0}$ \\
\hline
\end{tabular}

Table 3: Classification errors [\%] on the Speech database (Error without combination: $12.92 \%$ )

\begin{tabular}{|l||c|c|c|c|c|}
\hline & Set1 & Set2 & Set3 & Set4 & Set5 \\
\hline \hline$S A$ & 8.70 & 8.34 & 7.88 & 7.26 & 7.78 \\
\hline$W A$ & 7.56 & 7.64 & 7.64 & 7.06 & 7.68 \\
\hline$A H P_{1}$ & 6.84 & 7.26 & 7.04 & $\mathbf{6 . 7 6}$ & $\mathbf{7 . 4 8}$ \\
\hline$A H P_{2}$ & 6.74 & $\mathbf{6 . 9 0}$ & 6.98 & 6.82 & 7.56 \\
\hline$A H P_{3}$ & $\mathbf{6 . 6 7}$ & 6.94 & 6.96 & 6.80 & 7.58 \\
\hline$A H P_{4}$ & 6.78 & 7.00 & $\mathbf{6 . 8 8}$ & 6.82 & 7.54 \\
\hline
\end{tabular}

Table 4: Classification errors [\%] on the Letter database (Error without combination: 13.78\%)

\begin{tabular}{|l||r|r|r|r|r|}
\hline & Set1 & Set2 & Set3 & Set4 & Set5 \\
\hline \hline$S A$ & 10.95 & 10.35 & 10.35 & 10.00 & 10.05 \\
\hline$W A$ & 10.50 & 10.35 & 10.45 & 9.90 & 10.00 \\
\hline$A H P_{1}$ & 10.05 & 9.95 & 10.05 & 9.60 & $\mathbf{9 . 3 0}$ \\
\hline$A H P_{2}$ & 10.00 & 9.70 & $\mathbf{9 . 4 5}$ & 9.50 & 9.50 \\
\hline$A H P_{3}$ & $\mathbf{9 . 8 0}$ & $\mathbf{9 . 5 0}$ & 9.50 & $\mathbf{9 . 2 0}$ & 9.45 \\
\hline$A H P_{4}$ & 9.90 & 9.55 & 9.75 & 9.30 & 9.50 \\
\hline
\end{tabular}

Table 5: Classification errors [\%] on the Satimage database. (Error without combination: $12.05 \%$ )

Tables 3, 4, and 5 show the results of the experiments. Columns represent the various classifier sets, while rows show the classification errors measured using the selected combination of the corresponding classifier group.

As expected, all the combinations here improved the generalization performance of the simple classifier. In almost every case AHP-based combinations outperformed the weighed averaging combinations. However, in some cases $S A$ performed better than $W A$ and the $A H P$ combiners, showing that the strong assumptions of the method are not always satisfied (Fumera and Roli, 2003).

The performance of $A H P$ combiners depends on the size of testing set. When the test sets selected were too small, the measured accuracy values did not characterize the goodness of the classifiers, and yielded 
wrong combination results. Increasing the size, however, makes the consistency index $(C I)$ tend to zero, producing weights that tend to the values calculated by weighted averaging. Determining the optimal size of the test set requires further study.

When considering the sensitivity for the selection of different classifier subsets, the AHP-based combiner has a behavior similar to that of the $W A$ method, hence the optimal classifier set can be selected by methods available for the averaging combiners (Roli and Fumera, 2002).

Lastly, we should say that the AHP-based combination scheme is a good tool for making the solution of the classification problem more accurate and reliable.

\section{Conclusion}

In this paper we proposed a new linear combination method, and compared its performance with those of other combiners. As shown in the experiments, AHP-based combinations proved an effective generalization of the weighted averaging rule; they outperformed the other averaging methods in almost every case.

Finally, we should mention that resampling techniques may further improve the performance of linear combinations, hence it is worth investigating generalizations of the bootstrapped learners Bagging (Breiman, 1996) and Boosting (Freund, 1990) in future work.

\section{REFERENCES}

Breiman, L. (1996). Bagging predictors. Machine Learning, 24(2):123-140.

Duda, R. O., Hart, P. E., and Stork, D. G. (2001). Pattern Classification. John Wiley and Son, New York.

Freund (1990). Boosting a weak learning algorithm by majority. In Proceedings of the Workshop on Computational Learning Theory (COLT 1990). Morgan Kaufmann Publishers.

Fumera, G. and Roli, F. (2003). Linear combiners for classifier fusion: Some theoretical and experimental results. In 4th Int. Workshop on Multiple Classifier Systems (MCS 2003), Guildford, UK. Springer-Verlag, LNCS.

Jain, A. K., Duin, R. P. W., and Mao, J. (2000). Statistical pattern recognition: A review. IEEE Transactions on Pattern Analysis and Machine Intelligence, 22(1):4-37.
Kocsor, A. and Tóth, L. (2003). Kernel-based feature extraction with a speech technology application. In IEEE TRANS. ON SIGNAL PROCOCESSING.

Perrone, M. P. and Cooper, L. N. (1993). When networks disagree: Ensemble methods for hybrid neural networks. In Mammone, R. J., editor, Neural Networks for Speech and Image Processing, pages 126-142. Chapman-Hall.

Roli, F. and Fumera, G. (2002). Analysis of linear and order statistics combiners for fusion of imbalanced classifiers. In 3rd Int. Workshop on Multiple Classifier Systems (MCS 2002), Cagliari, Italy. Springer-Verlag, LNCS.

Saaty, T. L. (1980). The Analytic Hierarchy Process. McGraw-Hill, New York.

Tumer, K. and Ghosh, J. (1996). Analysis of decision boundaries in linearly combined neural classifiers. Pattern Recognition, 29:341-348.

Vapnik, V. N. (1998). Statistical Learning Theory. John Wiley and Son.

Xu, L., Krzyzak, A., and Suen, C. (1992). Method of combining multiple classifiers and their application to handwritten numeral recognition. IEEE Trans. on SMC, 22(3):418-435. 